\title{
Correction: Immunity and mental illness: findings from a Danish population-based immunogenetic study of seven psychiatric and neurodevelopmental disorder
}

\author{
Ron Nudel · Michael E. Benros · Morten Dybdahl Krebs • Rosa Lundbye Allesøe - Camilla Koldbæk Lemvigh • \\ Jonas Bybjerg-Grauholm • Anders D. Børglum - Mark J. Daly · Merete Nordentoft - Ole Mors · David M. Hougaard • \\ Preben Bo Mortensen · Alfonso Buil · Thomas Werge · Simon Rasmussen · Wesley K. Thompson
}

Published online: 8 December 2020

(c) The Author(s) 2020. This article is published with open access

Correction to: European Journal of Human Genetics https://doi.org/10.1038/s41431-019-0402-9

In the text of the original article linked to this correction article, we the authors include the ICD-10 codes for the diagnoses we used. We would also like to add the corresponding ICD-8 codes for some of the diagnoses, as a minority of the individuals might have received them while ICD-8 was being used in Denmark. This does not change the results or conclusions of the paper. However, for the sake of completeness, we believe the text should mention the codes that would have been used in those cases, in case the reader may need to look them up and/or compare them to studies which used ICD-8. Since the association with intellectual disability was the main new result from the paper, and some individuals received this diagnosis at a young age, unlike e.g. schizophrenia, they could have received an ICD-8 code (ICD-8 was used until 1994 and the first year of birth for the cohort is 1981), so it is important to note the codes used in those cases. We apologize for their omission from the original text. The original and revised text is as follows:

Original text (page 2 of the online PDF; page 1,446 of the printed PDF):

The phenotype groups included in this study were as follows: controls (no psychiatric or neurodevelopmental diagnoses, as per ICD-10 codes F00-F99); schizophrenia cases (F20); bipolar disorder (BPD) cases (F30-F31); single and recurrent depressive disorder cases (F32-F33); anorexia nervosa cases (F50.0); ASD cases (F84.0, F84.1, F84.5, F84.8, and F84.9), ADHD cases (F90.0) and intellectual disability (ID) cases (F70-F79).

Revised text:

The phenotype groups included in this study were as follows: controls (no psychiatric or neurodevelopmental diagnoses, as per ICD-10 codes F00-F99; equivalent ICD-8 codes 290-315); schizophrenia cases (F20; $295 . x 9$ excluding 295.79); bipolar disorder (BPD) cases (F30-F31; 296.19, 296.39, 298.19); single and recurrent depressive disorder cases (F32-F33; 296.09, 296.29, 298.09, 300.49); anorexia nervosa cases (F50.0; 306.50); ASD cases (F84.0, F84.1, F84.5, F84.8, and F84.9), ADHD cases (F90.0) and intellectual disability (ID) cases (F70-F79; 311.xx, 312.xx, 313.xx, 314.xx, 315.xx).

Open Access This article is licensed under a Creative Commons Attribution 4.0 International License, which permits use, sharing, adaptation, distribution and reproduction in any medium or format, as long as you give appropriate credit to the original author(s) and the source, provide a link to the Creative Commons license, and indicate if changes were made. The images or other third party material in this article are included in the article's Creative Commons license, unless indicated otherwise in a credit line to the material. If material is not included in the article's Creative Commons license and your intended use is not permitted by statutory regulation or exceeds the permitted use, you will need to obtain permission directly from the copyright holder. To view a copy of this license, visit http://creativecommons. org/licenses/by/4.0/. 